\title{
Comparative Study of Structural Parameters of Normal and Alkali Treated Polyester Fiber by SAXS, SEM, and Instron
}

\author{
Tankadhar PATEL ${ }^{\dagger}$ and Smrutiskha BAL \\ Department of Physics, Regional Engineering College, \\ Rourkela-769008 (Orissa), INDIA \\ (Received June 2, 2000; Accepted November 28, 2000)
}

\begin{abstract}
Physical and macromolecular parameters of normal and alkali treated polyester fiber have been investigated applying Small Angle X-Ray Scattering (SAXS) technique using the theories of Vonk [J. Appl. Crystallogr., 6, 81 (1973)] and Ruland [J. Appl. Crystallogr., 4, 70 (1971)] treating the fiber as non-ideal two-phase system. Intensity data have been collected using compact Kratky camera having counter attachment. The three- and one-dimensional correlation function have been computed from the background corrected SAXS profiles and parameters like the width of transition layer $(E)$, the average periodicity transverse to the layer $(D)$, the specific inner surface $(S / V)$, the length of coherence $\left(l_{\mathrm{c}}\right)$, the transversal lengths in matter and void $\left(\bar{l}_{1}\right.$ and $\left.\bar{l}_{2}\right)$, the range of inhomogenity $\left(l_{\mathrm{r}}\right)$, the volume fractions of matter and void $\left(\phi_{1}\right.$ and $\left.\phi_{2}\right)$, the volume fraction of transition layer $\left(2 E_{\mathrm{v}} / D\right)$ and characteristic number $\left(f_{\mathrm{c}}\right)$ have been determined. Further, fiber diameter, percentage elongation and Young's modulous, have been estimated from SEM and Instron respectively. The analysis of all the above parameters leads us to conclude that strong alkali $(\mathrm{NaOH})$ of $\mathrm{pH} 12$ have mild effect on the fiber at ambient temperature.
\end{abstract}

KEY WORDS Polyester Fiber / SAXS Technique / SEM / Non-Ideal Two-Phase System / Correlation

Function / Width of Transition Layer / Transverse Periodicity /

Polyester is a desirable synthetic fiber because of its strength, inertness and thermal stability, due to which it becomes useful for microelectronics and solid state devices along with textile applications. ${ }^{1}$ In our present work we have done SAXS study on normal and alkali treated polyester fibers to show effects of alkali by determining various micro and macromolecular parameters.

The work of Patel et al. ${ }^{2}$ on Nylon 6 shows that synthetic polymers possess non-ideal two-phase structure. The analysis of scattering data of polyester reveals that it belongs to non-ideal two-phase system.

Polymers often exhibit deviation from Porod ${ }^{3,4}$ law. The product $\check{I} p(s) \times s^{3}$ does not lead to a constant value at large $s(=2 \sin \theta / \lambda)$ but leads to either positive or negative deviation. Negative deviation ${ }^{5,6}$ has been observed in semi-crystalline polyester due to crystal surface irregularities and the existence of diffuse phase boundary causing depletion of intensity at high angles.

Ruland ${ }^{7}$ has modified Porod's law to determine density fluctuation and width of transition layer. Vonk ${ }^{8}$ has developed practical aspects of Ruland method, which have been followed in this work.

\section{EXPERIMENTAL}

\section{Sample}

The polyester fiber used for our investigations was obtained from a private firm. The method of preparation is as follows.

Polyester fiber is produced from poly(ethylene terephthalate), i.e., PET polymer. Ethylene glycol and purified terepthalic acid first undergoes esterification process followed by polycondensation process to produce PET (polyethylene terepthalate) chips. Then it undergoes drying, melt spinning etc. processes to produce polyester fibers. ${ }^{9,10,11,12}$ The chemical reaction for prepa- ration of $\mathrm{PET}$ is given below.

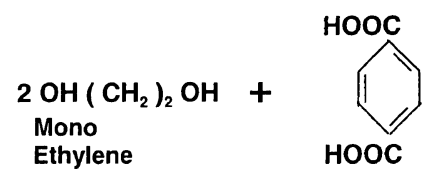

PTA ( Purified Terephthalic acid)

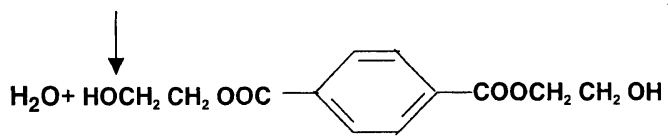

PET ( Polyethylene Terephthalate)

Polyester fibers interact with detergents, soaps etc. while in use. Moreover these fibers are also subjected to dry cleaning. Therefore it is important to understand the effects of alkalis and acids on these fibers. In this paper we make an attempt to investigate the effects of alkalis on polyester fiber.

\section{Experimental}

A part of the sample was soaked in Sodium Hydroxide solution at concentration $\mathrm{pH} 12$ for $48 \mathrm{~h}$ at ambient temperature and then it was washed with distilled water and dried.

SAXS intensity data for both untreated (here called sample-I) and treated sample with $\mathrm{NaOH}$ of $\mathrm{pH} 12$ (called sample-II) were collected using compact Kratky camera having counter attachment. A Phillips PW 1729 $\mathrm{X}$-Ray generator with a copper target operated at $35 \mathrm{kV}$ and $20 \mathrm{~mA}^{13}$ was used for collection of intensity data. The entrance and counter slit attached to the Kratky camera were adjusted at $80 \mu \mathrm{m}$ and $250 \mu \mathrm{m}$ respectively. The room temperature was maintained at $22.5 \pm 0.5^{\circ} \mathrm{C}$ and sample to counter distance was $20 \mathrm{~cm}$. Monochro-

${ }^{\dagger}$ To whom correspondence should be addressed (Fax: 91-661-571169, 91-661-572926, E-mail: tpatel@rec.ori.nic.in). 

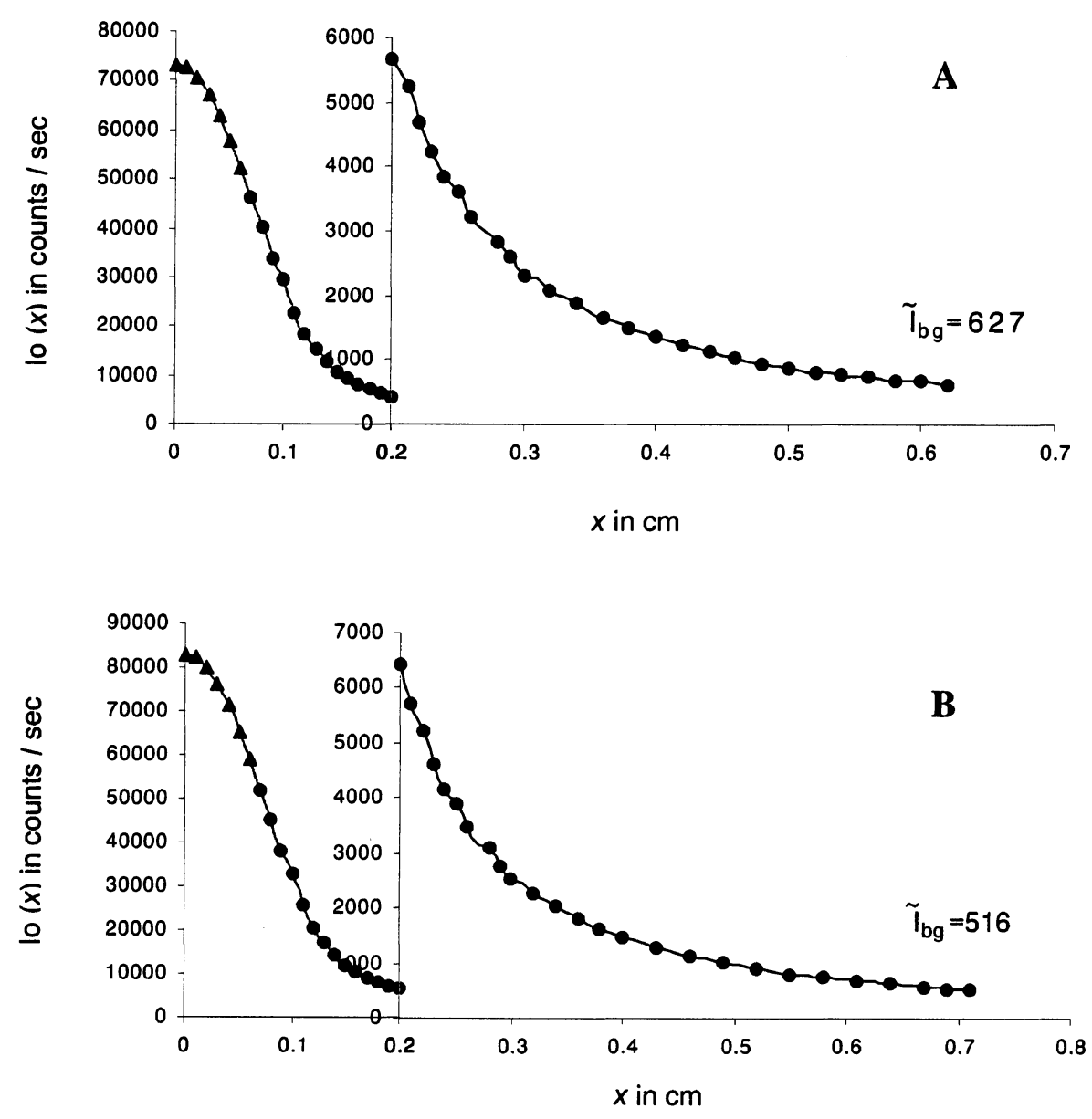

Figure 1. Smeared out scattering curve of sample-I and II are shown in (A) and (B) respectively.

matic $\mathrm{Cu}-K_{\alpha}(\lambda=1.54 \AA)$ radiation was obtained using a nickel filter of $10 \mu \mathrm{m}$ thickness which was used to irradiate polyester fibers packed in Mark capillary tube of 1 $\mathrm{mm}$ diameter. The intensity data have been collected keeping the sample both in parallel and perpendicular positions to the X-Ray beam for testing its isotropic nature. To avoid parasitic scattering by air present between sample and detector the irradiation was carried out under a vacuum of approximately $(1 / 2) m \mathrm{bar}^{14}$ The smeared out intensity curve (Figure 1) was plotted by subtracting blank capillary data from observed scattering data. Constant background intensity has been subtracted from observed data according to Konrad and Zachmann ${ }^{15}$ for the purpose of estimation of different parameters of the samples.

Young's modulus and percentage elongation of the samples were found out employing "Instron Tensile Tester Model-1026" at Physics Deptt, UDCT, Mumbai. Furthermore, SEM study was carried out using "Scanning Electron Microscope-JEOL JSM 840 A" at Advanced Center for Material Science (ACMS), IIT Kanpur.

\section{THEORY}

Theories of Vonk ${ }^{8}$ and Ruland ${ }^{7}$ have been applied here to calculate various macromolecular parameters, treating the sample as a non-ideal two-phase system having isotropic structure. An important parameter in charac- terization of structure is given by

$$
R=\frac{\left\langle|\operatorname{grad} \eta|^{2}\right\rangle}{\left\langle\eta^{2}\right\rangle}=4 \pi^{2} \frac{\int_{0}^{\infty} s^{4} I(s) \mathrm{d} s}{\int_{0}^{\infty} s^{2} I(s) \mathrm{d} s}=6 \pi^{2} \frac{\int_{0}^{\infty} s^{3} \check{I}(s) \mathrm{d} s}{\int_{0}^{\infty} s \tilde{I}(s) \mathrm{d} s}
$$

where $I(s)$ and $I(s)$ are desmeared and smeared out intensities respectively in arbitrary units, $\eta$ is deviation of electron density from its mean value, $s$ is a vector in a reciprocal (Fourier) space. In an ideal two-phase system, the gradient at phase boundary is infinity and $R$ also goes to infinity. The case where the minimum value of $R$ $=4 \pi^{2} s_{0}^{2}$ corresponds to the intensity function $I(s)$ consisting of a single peak at $s=s_{0}$. Such an intensity function is obtained from a randomized layer structure. Here electron density perpendicular to layers fluctuates according to a sine function.

Further $R$ has been expressed in terms of intensity function $I(x)$ by T. Patel et al. ${ }^{16,17}$ as

$$
R=3 / 2(2 \pi / \lambda a)^{2} \int_{0}^{\infty} x^{3} \tilde{I}(x) \mathrm{d} x / \int_{0}^{\infty} x \tilde{I}(x) \mathrm{d} x
$$

where $x$ is the position coordinate of the scattered intensity from the center of primary beam, a, distance of sample from the counter slit and $s=2 \theta / \lambda=x / \lambda a, 2 \theta$ is the scattering angle. Small, positive, and finite value of $R$ 
suggests that corrugation at the phase boundary exists and electron density changes from matter phase to void phase over a transition layer $E$ and in consequence Porod's law, fails.

The width of transition layer $E$ can be obtained from the relation derived by $\mathrm{Vonk}^{8}$

$$
E=(-4 / R)(\mathrm{d} C(r) / \mathrm{d} r)_{r=E v}
$$

where $C(r)$ is the three-dimensional correlation function of a sample normalized to unity at the origin in real space. The function $C(r)$ was derived by Mering and Tchoubar $^{18}$ and expressed in terms of ' $x$ ' by T. Patel $e t$ al. ${ }^{17}$ as

$$
C(r)=\int_{0}^{\infty} x \check{I}(x) J_{0}(2 \pi r x / \lambda a) \mathrm{d} x / \int_{0}^{\infty} x \check{I}(x) \mathrm{d} x
$$

$J_{0}$ is Bessel function of zero order of the first kind. According to Vonk ${ }^{8}$ for an isotropic sample

$$
R=-3\left(\mathrm{~d}^{2} C(r) / \mathrm{d} r^{2}\right)_{r=0}
$$

However whenever $R$ is determined from the correlation function care must be taken to avoid experimental error at the tail of the scattering curve as the correlation function in the origin is very sensitive to such error as pointed out by Caulfield and Ullman. ${ }^{19}$

One- dimensional correlation function $C_{1}(y)$ given by Kortleve and Vonk ${ }^{20}$ for layer structure in terms of $x^{17}$ is expressed as

$$
C_{1}(y)=\int_{0}^{\infty} x \bar{I}(x)\left[J_{0}(z)-z J_{1}(z)\right] \mathrm{d} x / \int_{0}^{\infty} x \bar{I}(x) \mathrm{d} x
$$

where $z=2 \pi x y / \lambda a$ and $J_{1}$ is the Bessel function of first order and of the first kind. According to Vonk $^{8}$ the position of first subsidiary maximum in the one-dimensional correlation function $C_{1}(y)$, gives the value of the average periodicity $D$ transverse to the layers.

The specific inner surface defined as the phase boundary per unit volume of the dispersed phase is.

$$
(S / V)=2 / D
$$

The relation

$$
\left\langle\eta^{2}\right\rangle\langle\Delta \eta\rangle^{2}=\phi_{1} \phi_{2}-(E S / 6 V)
$$

was given by Vonk where $\phi_{1}, \phi_{2}$ are the volume fraction of matter and void region respectively and the relation $\phi_{1}+\phi_{2}=1$ can be used to determine the individual values of $\phi_{1}$ and $\phi_{2}$.

As defined by Mittelbach and Porod ${ }^{21}$ for a two-phase system transversal lengths of matter and void phases for a three-dimensional system are given by the relation

$$
\bar{l}_{1}=4 \phi_{1}(V / S), \bar{l}_{2}=4 \phi_{2}(V / S)
$$

and

$$
1 / \bar{l}_{\mathrm{r}}=1 / \bar{l}_{1}+1 / \bar{l}_{2}
$$

where $\bar{l}_{\mathrm{r}}$ is the range of inhomogenity. Moreover,

$$
l_{\mathrm{c}}=2 \int_{0}^{\infty} C(r) \mathrm{d} r
$$

$l_{\mathrm{c}}$ being the length of coherence which is the average value of length of cords drawn in all directions. A second method to determine the width of transition layer $E$ as given by Ruland ${ }^{7}$ for a non-ideal two-phase system and expressed in terms of $x$ is given by

$$
\tilde{I}(x) \times x=\pi C / 2 \times(\lambda a)^{3} \times x^{-2}-\pi^{3} C / 3 \times(\lambda a) \times E^{2}
$$

From the above equation the plot of $\tilde{I}(x) x$ vs. $x^{-2}$ at the tail end of the SAXS pattern is known as Ruland plot. Equation 12 reduces to Porod's law if $E=0$, for an ideal two-phase system.

\section{RESULTS AND DISCUSSION}

Five intensity values $\tilde{I}(x)$ near the origin were fitted to a Gussian curve $(x)$.

$$
\check{I}(x \rightarrow 0)=p \times \exp \left(-q x^{2}\right)
$$

by least square technique. The values of $p$ for sample I and II were obtained as 73339 and $83004.6 \mathrm{~J} \mathrm{~m}^{-2} \mathrm{~s}^{-1}$ respectively and corresponding values of $q$ as 97.29 and $97.3 \mathrm{~m}^{-2}$ respectively. The scattering curves were extrapolated to zero angle by using values of $p$ and $q$ (Figure 1) indicating the extrapolated points by symbol $\Delta$. The method of extrapolation has very little effect on the relevant part of the correlation functions, i.e., position and height of first subsidiary maximum of the onedimensional correlation function is not affected much. ${ }^{17}$ Values of $R$ were determined using eq 2 and were found to be $(23.12 \pm 0.28) 10^{-4}$ and $(28.54 \pm 0.33) 10^{-4} \AA^{-2}$ for sample-I and sample-II respectively. Small and positive values of $R$ parameter for both samples prove that electron density gradient at phase boundary is finite thus suggesting belongingness of sample to a non-ideal twophase system. Values of $R$ obtained by relation eq 5 using five-point forward difference method were found out to be $(23.12 \pm 0.03) \times 10^{-4}$ and $(28.82 \pm 0.008) \times 10^{-4}$ $\AA^{-2}$ for sample-I and sample-II respectively. These figures are almost equal to the values of $R$ of the corresponding samples that were already obtained using eq 2 , which indicates isotropic nature of the sample. The symmetric intensity pattern in Figure 2 also indicates the isotropic nature of the sample. The difference of intensity corresponding to the two types of mounting of sample is due to the difference in quantity of sample exposed. ${ }^{22,23}$

For various values of $r$ and $y$, the three- and onedimensional correlation function for both samples were computed respectively and are shown in Figures 3 and 4 with error bars. According to Vonk the width of transition layer $(E)$ was obtained from the plot of $\left(-\frac{4}{R}\right) \times$ $\left[\frac{\mathrm{d} C(r)}{\mathrm{d} r}\right] v s . r$ in Figure 5.

The value of average periodicity transverse to the layers $(D)$ were obtained from the position of first subsidiary maximum in one-dimensional correlation function (Figure 4). The value of the specific inner surface $(S / V)$, 


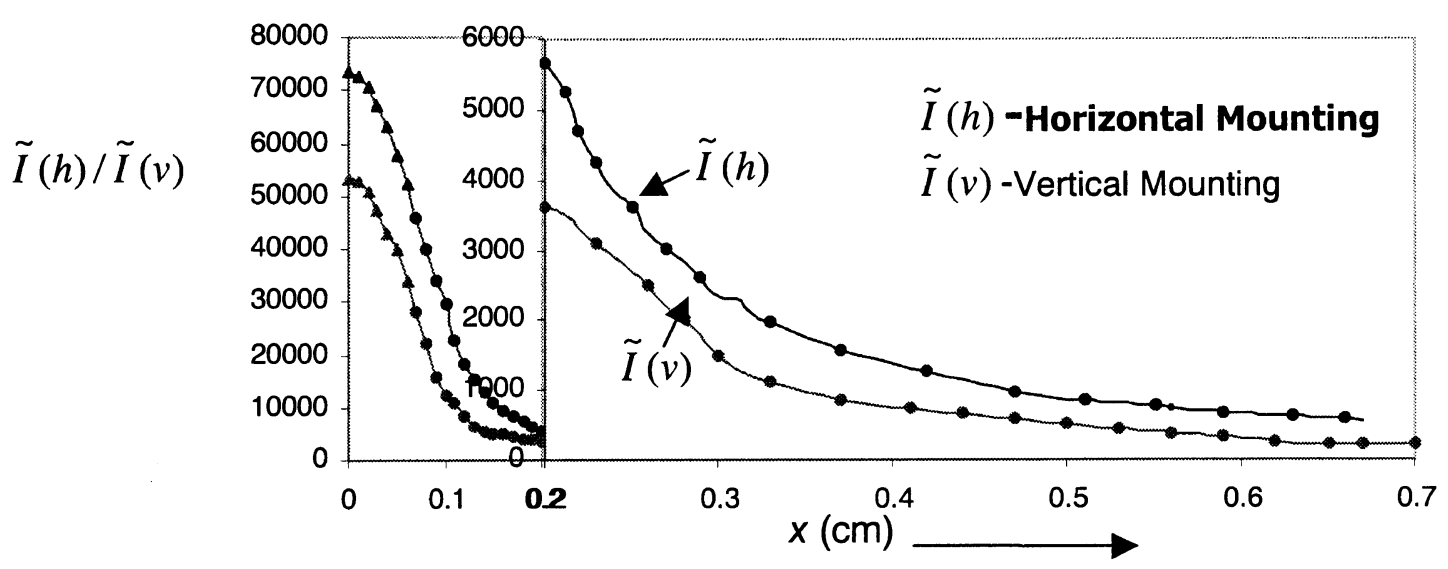

Figure 2. Smeared out scattering profiles of sample-I and II extrapolated points are shown by $\Delta$ sign.

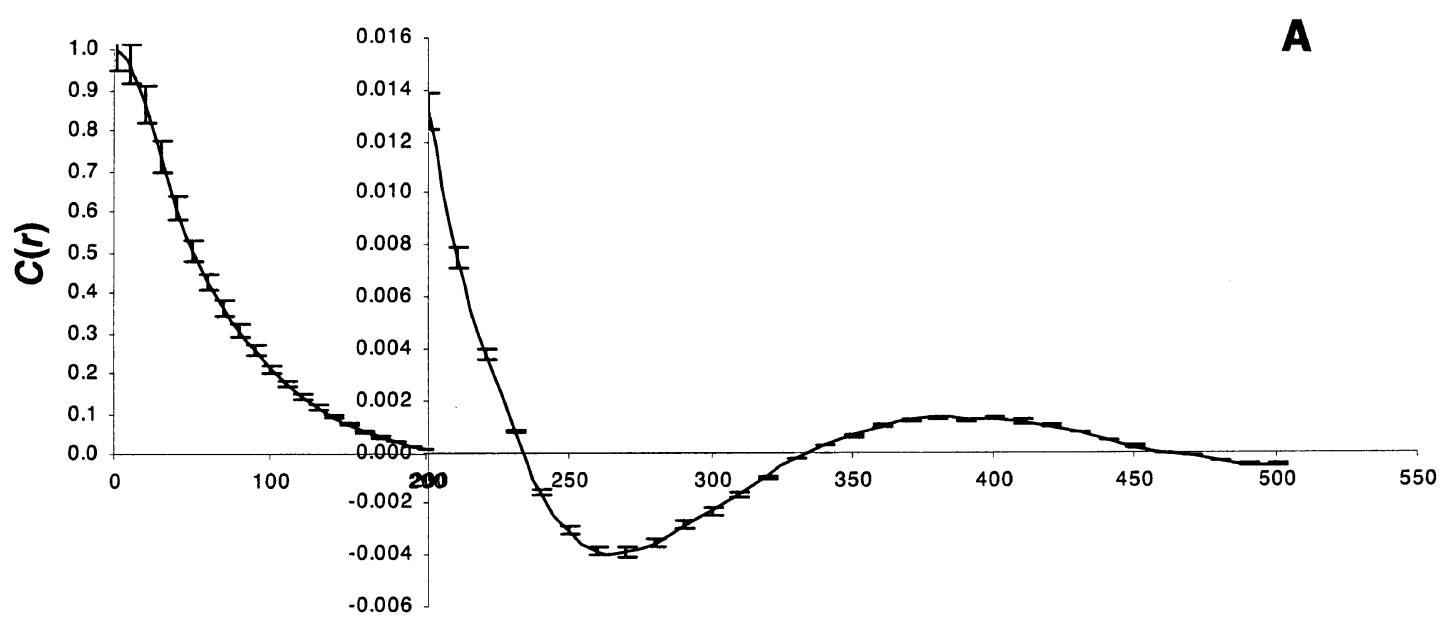

$r(\AA)$

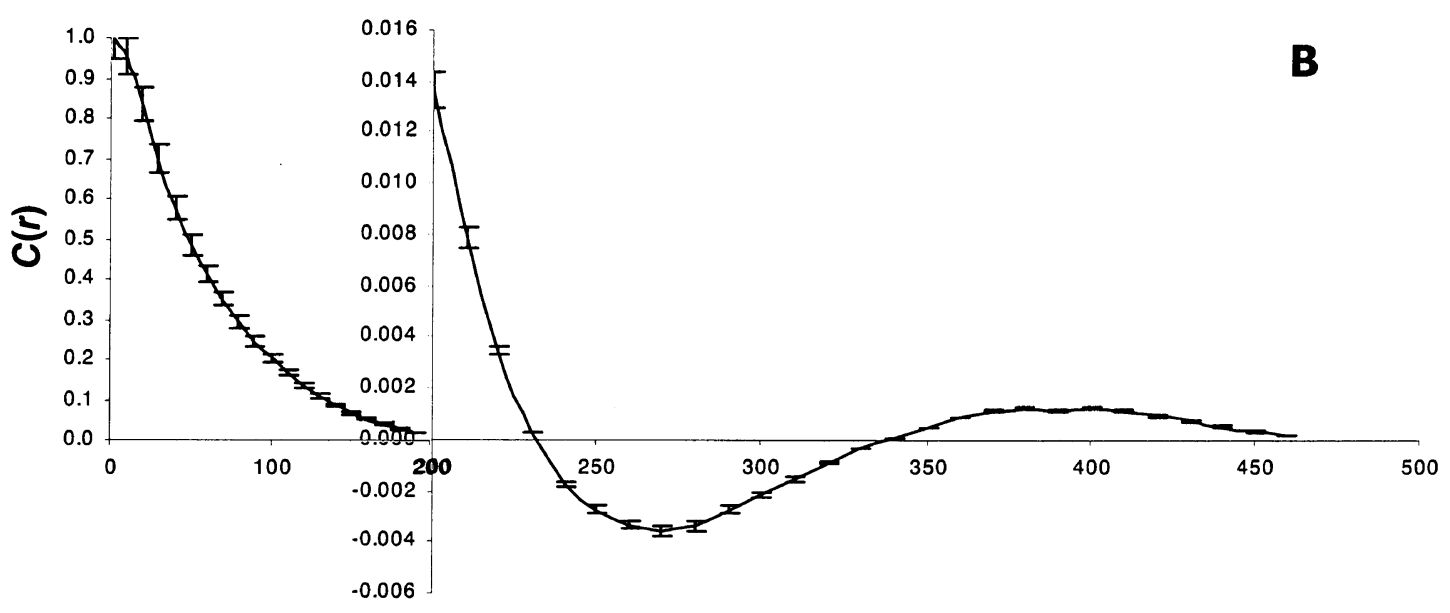

$r(\AA)$

Figure 3. Three dimensional correlation function " $C(r)$ " against " $r$ " with error bars of sample-I (Figure A) and II (Figure B).

the volume fractions of matter and void $\left(\phi_{1}\right.$ and $\left.\phi_{2}\right)$, the transversal lengths in matter and void $\left(\bar{l}_{1}\right.$ and $\left.\bar{l}_{2}\right)$, range of inhomogenity $\left(l_{\mathrm{r}}\right)$, the length of coherence $\left(l_{\mathrm{c}}\right)$ were estimated. All main SAXS parameters are tabulated in Table I.
Ruland Plot $\tilde{I}(x) x$ vs. $x^{-2}$ of the two samples are shown in Figure 6 which give straight lines at the limiting region of the scattering curve where slopes are $(98 \pm$ $0.4),(151.32 \pm 0.4)$ and $y$ intercepts are $(-248.99 \pm 1)$, $(-333.96 \pm 1.7)$ for samples I and II respectively. Using 

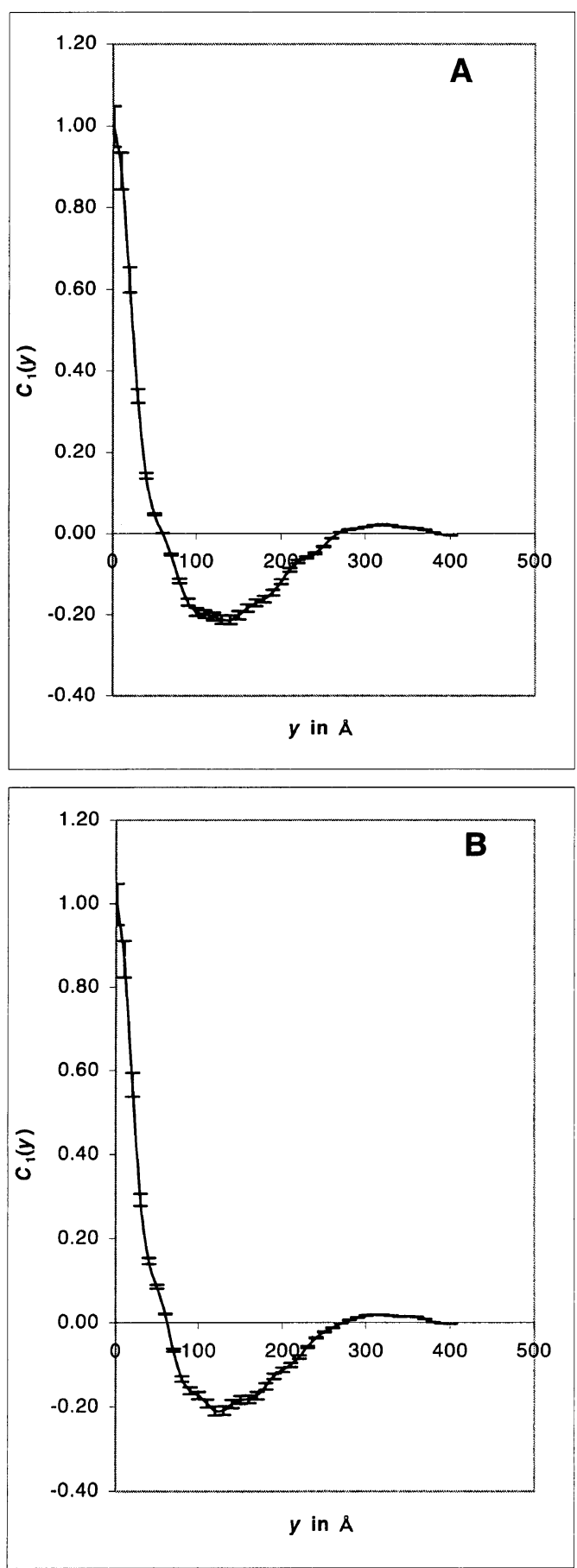

Figure 4. Curves of one-dimensional correlation function " $C_{1}(y)$ " against " $y$ " with error bars of sample-I(A) and II(B).

these slopes and intercepts, the width of transition layer $\left(E_{\mathrm{R}}\right)$ by Ruland method were found out which is quite close to $E_{\mathrm{v}}$ (Table I). Standard deviation of the intensities $\sigma(I)^{1 / 2}$ was calculated at tail region of SAXS curve of samples and values are well within the permissible lim$\operatorname{its}^{24}(0.5)$ (Table I). The regression coefficient $\gamma$ for sample-I $=0.91$ and for sample-II $=0.9$ confirms the accuracy of collected data. ${ }^{25}$ Lamellar models for the two samples are shown in Figure 7 which are based on findings of SAXS parameters (Table I).

The average values of diameters of sample-I and sample-II obtained from SEM study are $2069 \AA$ and
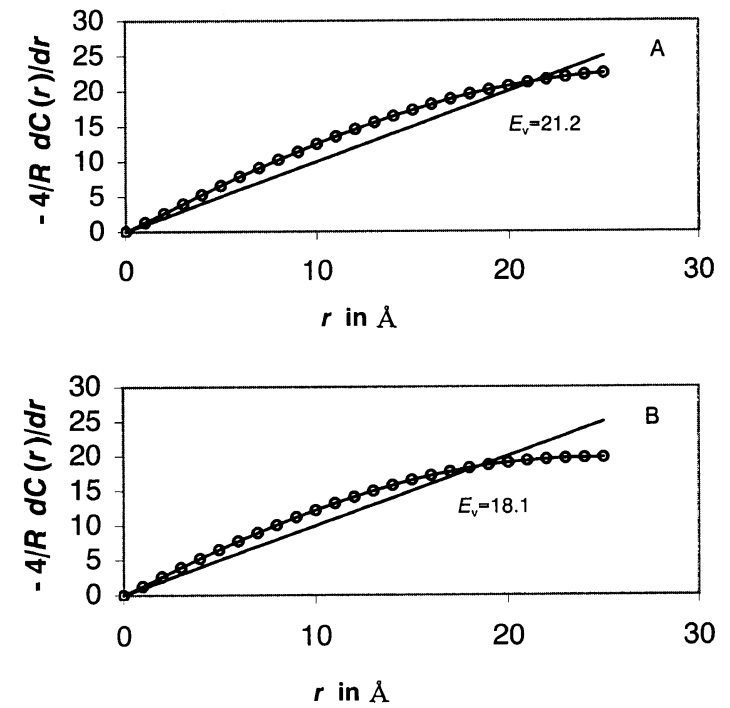

Figure 5. Curves of " $-4 R(\mathrm{~d} C(r) / \mathrm{d} r)$ " against " $r$ " of sample-I in (A) and II in (B).

Table I. Macromolecular parameters of polyester fiber by SAXS

\begin{tabular}{|c|c|c|}
\hline Parameters & Sample-I & Sample-II \\
\hline$D / \AA$ & 319 & 314 \\
\hline$E_{\mathrm{v}} / \AA$ & $21.2 \pm 0.017$ & $18.1 \pm 0.017$ \\
\hline$E_{\mathrm{R}} / \AA$ & $19.13 \pm 0.04$ & $17.8 \pm 0.05$ \\
\hline$\frac{S}{V} / \AA^{-1}$ & 0.006 & 0.0064 \\
\hline$\phi_{1} / \%$ & $0.84641 \pm 0.00003$ & $0.82 \pm 0.000025$ \\
\hline $\begin{array}{c}\phi_{2} / \% \\
2 E_{\mathrm{V}}\end{array}$ & $0.1536 \pm 0.00003$ & $0.18 \pm 0.000025$ \\
\hline$D$ & $13.29 \pm 0.0106$ & $11.5 \pm 0.0109$ \\
\hline$l_{1} / \AA$ & $540 \pm 0.019$ & $514.96 \pm 0.0155$ \\
\hline$l_{2} / \AA$ & $97.99 \pm 0.019$ & $113.04 \pm 0.0155$ \\
\hline$l_{\mathrm{r}} / \AA$ & $82.94 \pm 0.011$ & $94.2 \pm 0.011$ \\
\hline$l_{\mathrm{c}} / \AA$ & $127.71 \pm 0.22$ & $123.34 \pm 0.1$ \\
\hline$\sigma$ & 0.16 & 0.12 \\
\hline$\gamma$ & 0.91 & 0.9 \\
\hline
\end{tabular}

$1993 \AA$ respectively. The SEM micrographs of both samples at different magnifications are shown in Figure 8. Each SEM photograph contains a dark and bright region clearly indicating the two-phase nature of the sample. Moreover the photographs at 5000 magnification clearly show that there is no preferred orientation of the matter in any direction confirming isotropic nature of the sample.

Tensile study shows that percentage elongation of sample-I and sample-II are $20 \%$ and $28 \%$ and Young's moduli of the same are $3.9 \times 10^{9} \mathrm{~N} \mathrm{~m}^{-2}$ and $2.8 \times 10^{9}$ $\mathrm{N} \mathrm{m}^{-2}$ respectively.

\section{CONCLUSIONS}

The values of $C(r)$ show damped oscillatory behavior at large values of $r^{17,26}$ for non-ideal two-phase system and this trend is evident for both the samples. Negative intercepts of Ruland plot confirm the non-ideal twophase nature. The values of width of transition layer $E_{\mathrm{v}}$ and $E_{\mathrm{R}}$ obtained by the methods of Vonk $\left(E_{\mathrm{v}}\right)$ and Ruland $\left(E_{\mathrm{R}}\right)$ are nearly equal confirming the correctness of collected data and method of analysis. The slight in- 

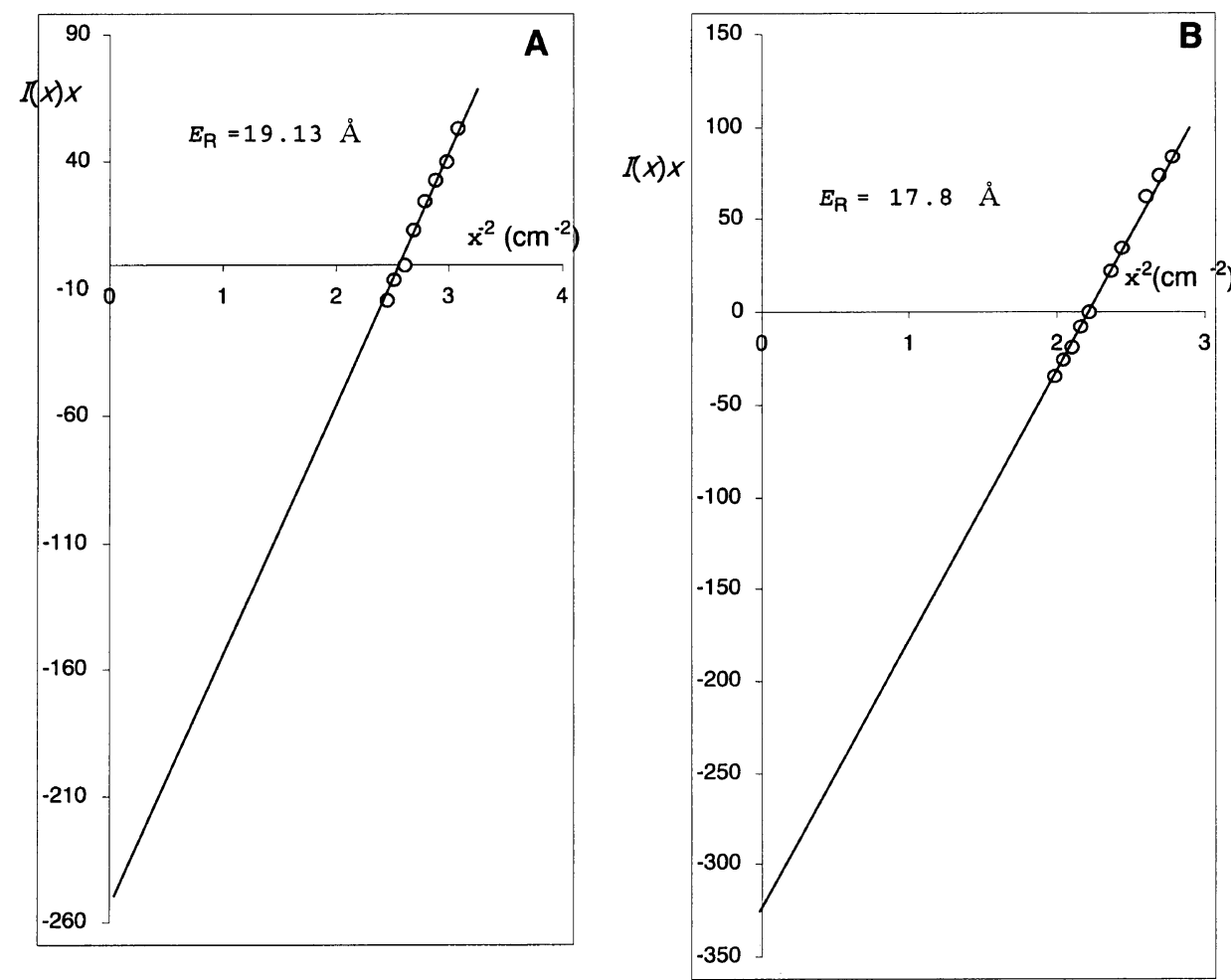

Figure 6. Ruland Plot of $I(x) x$ against $x^{2}$ of sample-I(A) and II(B).

$100 \AA$ $\rightarrow 1.28 \mathrm{~cm} /$

(A)

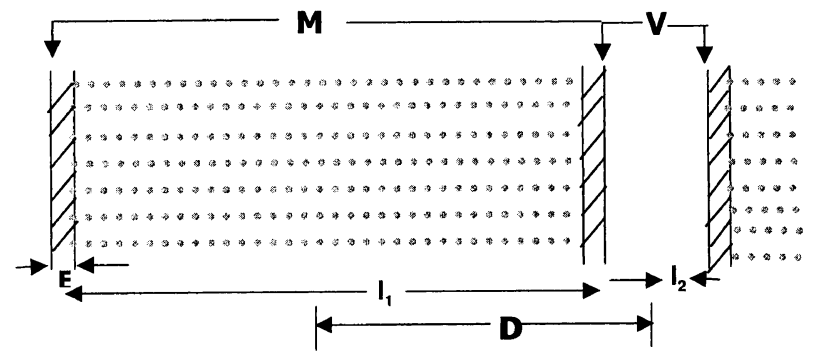

(B)

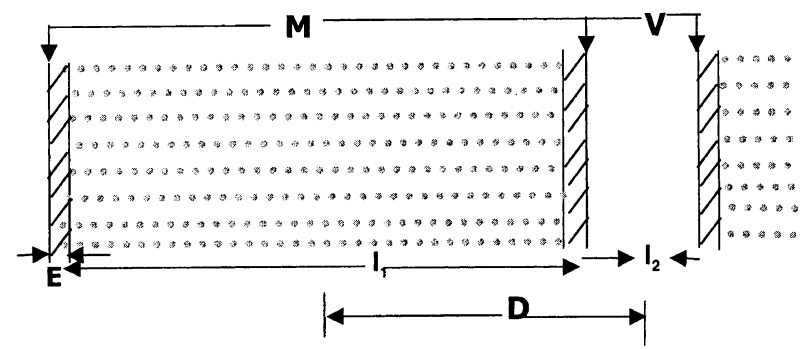

Figure 7. Lamellar model drawn to the scale $(100 \AA=1.28 \mathrm{~cm})$ of Sample-I (Figure A) and Sample-II (Figure B): $M$, matter; $V$, void; $D$, periodicity transverse to layer; $E$, width of the transition layer.

crease in the value of specific inner surface $(S / V)$ and decrease in the value of $D$ of sample-II as compared to corresponding parameters of original sample (Table I) shows that alkali treatment has mild effect on the sample. The value of $\phi_{1}$ and $l_{1}$ of sample-II decrease and that of $\phi_{2}$ and $l_{2}$ increase as compared to corresponding parameters of original sample (Table I) indicating that the macromolecules of the fiber break (degrade) at higher $\mathrm{pH}$ value of Sodium Hydroxide solution ( $\mathrm{pH} \mathrm{12).} \mathrm{Work}$ on this line is in progress subjecting samples with different $\mathrm{pH}$ values at various physical and chemical conditions.

The values of the diameters of sample-I and sample-II obtained from SEM study show that there is slight reduction in the diameter of the fiber when treated with alkali of higher $\mathrm{pH}$ ( $\mathrm{pH}$ 12). Increase in percentage elongation and decrease in Young's modulus of the fiber in alkali treated sample (sample-II) confirms to the findings obtained by SAXS that it undergoes degradation hence weakening.

\section{REFERENCES}

1. N. V. Bhat and S. G. Naik, Text. Res. J., 54, 868 (1984).

2. T. Mishra, S. S. Khuntia, V. H. Buch, and T. Patel, Polym. J., 24, 323 (1992).

3. G. Porod, Kolloid Z, 124, 83 (1951).

4. G. Porod, Kolloid Z., 125, 51 (1952).

5. E. Helfand, Acc. Chem. Res., 8, 295 (1975).

6. E. Helfand and Y. Tagami, Polym. Lett., 9, 741 (1971).

7. W. Ruland, J. Appl. Crystallogr., 4, 70 (1971).

8. C. G. Vonk, J. Appl. Crystallogr., 6, 81 (1973)

9. S. K. Pal, R. S. Gandhi, and V. K. Kothari, Tex. Res. J., 63, 71 (1993).

10. A. A. Vaidya, "Production of Synthetic Fibers", Prentice Hall of India, New-Delhi, 1988, p 34.

11. J. R. Whinfield, J. T. Dickson, W. K. Birtwistle, and C. G. Ritchie, "Chemistry of Terylene", Nature, 158, 930 (1946).

12. F. W. Billmeyer, Jr., "Textbook of Polymer Science", Wiley Interscience, New York, N.Y., 1971, p 413.

13. O. Glatter and O. Kratky, "Small Angle X-Ray Scattering", 


\section{SAMPLE-I (Original) SAMPLE-II (Alkali Treated)}
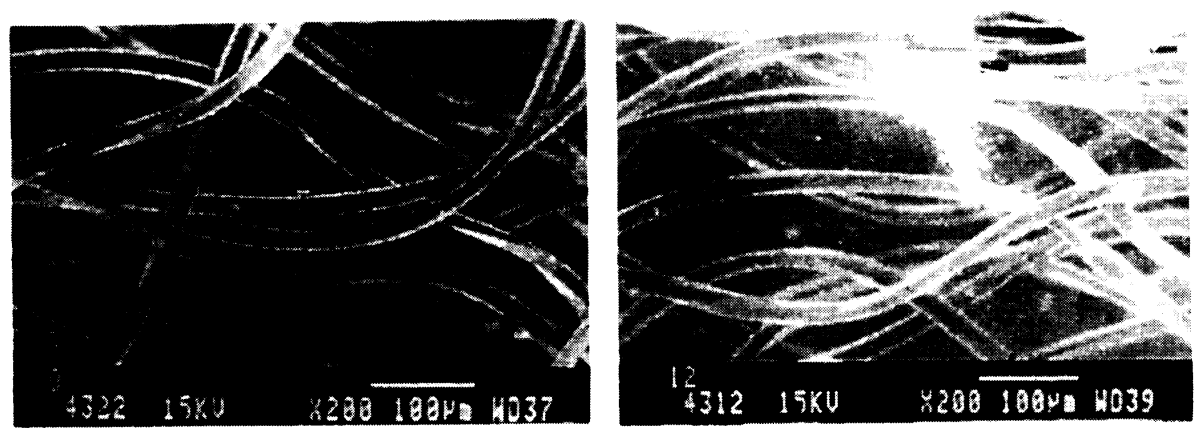

1(a)

2(a)
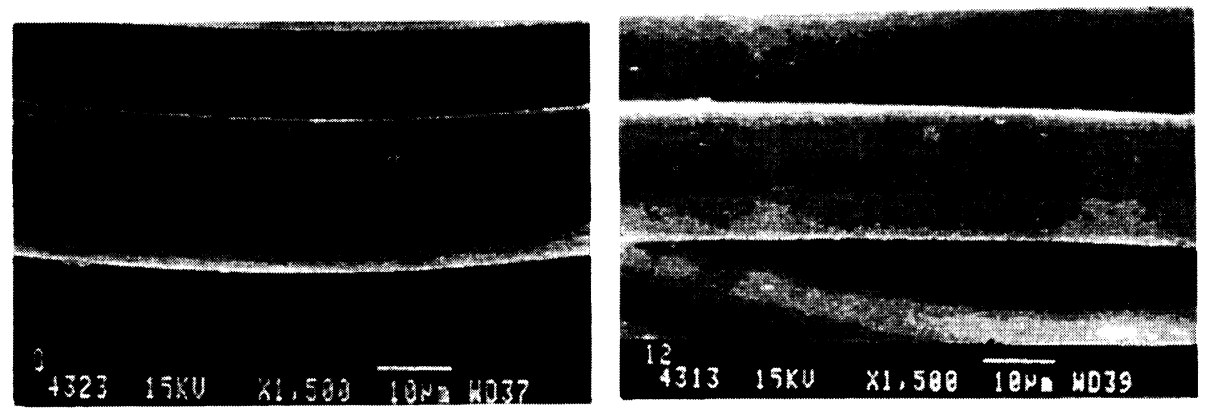

1 (b)

2(b)

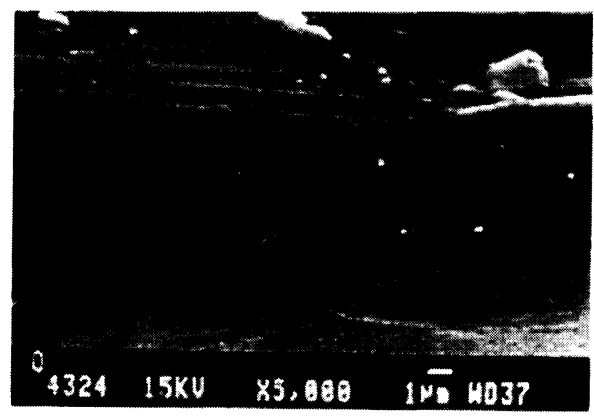

1 (c)

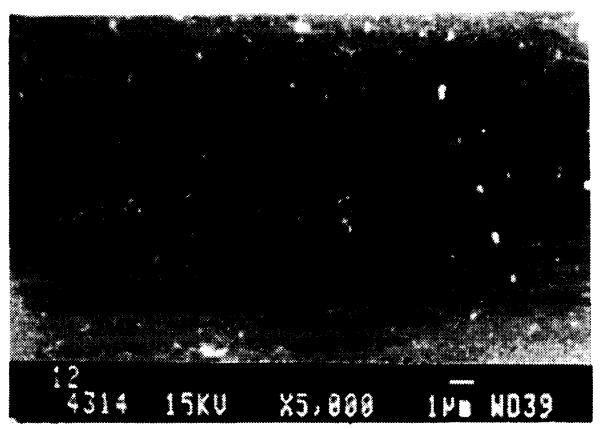

2(c)

Figure 8. SEM micrographs of SAMPLE-I (shown in 1(a), 1(b), and 1(c)) and SAMPLE-II (shown in 2(a), 2(b), and 2(c)) at different magnifications.

Academic Press, New York, N.Y., 1982, p 435.

14. R. W. Hendricks, J. Appl. Crystallogr., 3, 348 (1970).

15. G. Konrad and N. G. Zachmann, Kolloid Z. Z. Polym., 247, 851 (1971).

16. T. Mishra, D. K. Bisoi, T. Patel, K. C. Patra, and A. Patel, Polym. J., 20, 739 (1988).

17. T. Mishra, K. C. Patra, and T. Patel, Colloid Polym. Sci., 262, 611 (1984).

18. J. Mering and D. Tchoubar, J. Appl. Crystallogr., 1, 153 (1968).

19. D. Caulfield and R. Ullman, J. Appl. Phys., 33, 1737 (1962).

20. G. Kortleve and C. G. Vonk, Kolloid Z. Z. Polym., 225, 124 (1968).
21. P. Mittelbach and G. Porod, Kolloid Z. Z. Polym., 202, 40 (1965).

22. T. Mishra, P. Panda, T. Patel, D. K. Bisoi, and D. K. Panda, Jpn. J. Appl. Phys., 32, 4615 (1993).

23. T. Mishra, J. Shukla, Md. N. Khan, D. K. Bisoyi, and T. Patel, Polym. J., 29, 1007 (1997).

24. C. G. Vonk, J. Appl. Crystallogr., 4, 340 (1971).

25. J. Topping, "Errors of Observation and Their Treatment" 4th ed, Chapman and Hall (London), London, 1972, p 82 and p 104.

26. T. Patel and S. Bal, Bulletin of Orissa Physical Society, VIII, $74(2000)$. 